\title{
Dynamic modeling and sensitivity analysis of dAFM in the transient and steady state motions
}

Farokh Payam, A. (2016). Dynamic modeling and sensitivity analysis of dAFM in the transient and steady state motions. Ultramicroscopy, 169. https://doi.org/10.1016/j.ultramic.2016.05.011

Link to publication record in Ulster University Research Portal

\section{Published in:}

Ultramicroscopy

Publication Status:

Published (in print/issue): 04/07/2016

DOI:

10.1016/j.ultramic.2016.05.011

\section{Document Version}

Author Accepted version

\section{General rights}

Copyright for the publications made accessible via Ulster University's Research Portal is retained by the author(s) and / or other copyright owners and it is a condition of accessing these publications that users recognise and abide by the legal requirements associated with these rights.

\section{Take down policy}

The Research Portal is Ulster University's institutional repository that provides access to Ulster's research outputs. Every effort has been made to ensure that content in the Research Portal does not infringe any person's rights, or applicable UK laws. If you discover content in the Research Portal that you believe breaches copyright or violates any law, please contact pure-support@ulster.ac.uk. 


\title{
Dynamic Modeling and Sensitivity Analysis of dAFM in the Transient and Steady State Motions
}

\author{
Amir Farokh Payam \\ Instituto de Ciencia de Materiales de Madrid (ICMM), CSIC, Madrid, Spain
}

amir.farokh@csic.es

\begin{abstract}
In this paper, based on the slow time varying function theory, dynamical equations for the amplitude and phase of the dynamic atomic force microscope is derived. Then the sensitivity of the amplitude and phase to the dissipative and conservative parts of interaction force are investigated. The most advantage of this dynamical model is the ability to simulate and analysis the dynamics behavior of amplitude and phase of the AFM tip motion not only in the steady state but also in the transient regime. Using numerical analysis the transient and steady state behavior of amplitude and phase are studied and the sensitivity of amplitude and phase to the interaction force are analyzed.
\end{abstract}

Keywords- Slow time varying function, Amplitude, Phase, AFM, Dissipation, Virial

\section{Introduction}

Amplitude modulation atomic force microscopy has a significant ability of imaging surface topography of samples on an atomic scale. Analysis of the AM-AFM shows complicated relationship between the parameters of probe dynamics and the interaction force. Several theoretical models and expressions have been proposed to investigate this problem [1]-[26]. All of the methods have been used for the analysis of AM-AFM are concentrated on the steady state motion of the cantilever. Although all of these efforts are valuable and very informative, a theoretical approach towards study the dynamics of amplitude and phase of the AM-AFM especially in transient regime is in demand. Santos et al. [27]-[28] used the transient processes occurring in the tip-sample junction to reconstruct the force. Moreover, in [29]-[31], the transient motion of cantilever signal is used for the control of AFM. Also, using the transition from transient part to steady state regime, in [32]-[33] a new algorithm for high speed imaging of biopolymers using AFM is developed. However, study the amplitude and phase behavior in the transient regime needs more deep investigation. The interaction between tip and the sample composes of dissipative and conservative terms. Study the sensitivity of oscillating probe to the tip-sample interaction maybe useful to understand the resolution of imaging in AFM [11]-[16], [34]-[38]. Mechanical and chemical information about samples and atomic and/or nanoscale processes can be obtained from analysis of conservative and dissipative parts of interaction force [39]-[40]. Extracting such information enhances motivations behind the recent developments in AAFM techniques [41]-[45].

For this purpose, in this paper based on the slow time varying function theory [46]-[49], the dynamic equation of amplitude and phase are derived and the dynamic behavior of amplitude and phase not only in the steady state but also in the transient regime of operation is investigated. Moreover, based on the dynamics equations of AFM, the analytical expressions for the sensitivity of amplitude and phase to the dissipative and conservative parts of interaction force are derived. Finally using numerical analysis the effect of free amplitude, set-point amplitude, viscosity and stiffness on the sensitivity are investigated.

\section{Dynamics equation of AM-AFM}

The cantilever-tip motion in dynamic AFM (Fig. 1) is approximately described by:

$$
\ddot{z}+\frac{\omega_{0}}{Q} \dot{z}+\omega_{0}^{2} z=\frac{\omega_{0}^{2} F_{t s}}{k}+\frac{\omega_{0}^{2} F_{d}}{k}
$$

Where $\omega_{0}, k$ and $Q$ are resonance frequency, spring constant and quality factor, respectively. $F_{t s}$ is the interaction force between tip and the sample. 


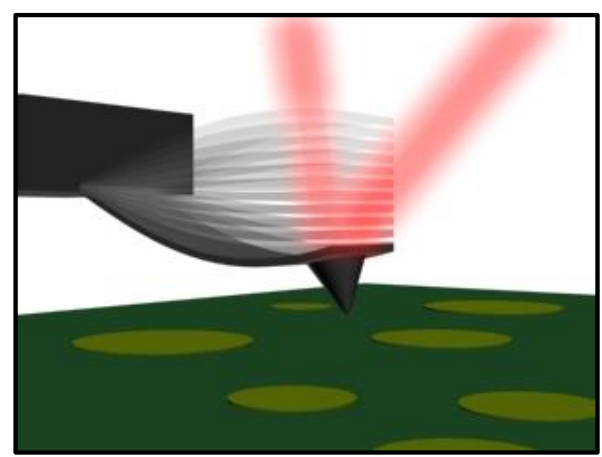

Fig.1. Scheme of tapping AFM

Assume that the solution of the above equation is represented as:

$z=A \cos (\omega t-\phi)$

where the amplitude $A$ and the phase $\phi$ are slowly varying functions of time and drive force is represented by:

$F_{d}=\frac{A_{0} k}{Q} \cos (\omega t)=F_{0} \cos (\omega t)$

Defining $\beta=\omega t-\phi$, the time derivative of the displacement is:

$\dot{z}=\dot{A} \cos (\beta)-A \omega \sin (\beta)+A \dot{\phi} \sin (\beta)=-A \omega \sin (\beta)$

Based on Eq. (4), the following condition is imposed:

$\dot{A} \cos (\beta)=-A \dot{\phi} \sin (\beta)$

By differentiating Eq. (4) with respect to time, the tip acceleration is obtained as:

$\ddot{z}=-\dot{A} \omega \sin (\beta)-A \omega^{2} \cos (\beta)+A \omega \dot{\phi} \cos (\beta)$

Substituting Eqs. (6) and (4) into Eq. (1) yields:

$-\dot{A} \omega \sin (\beta)-A \omega^{2} \cos (\beta)+A \omega \dot{\phi} \cos (\beta)-\frac{A \omega_{0} \omega \sin (\beta)}{Q}+A \omega_{0}^{2} \cos (\beta)=\frac{\omega_{0}^{2}}{k} F_{t s}+\frac{\omega_{0}^{2}}{k} F_{d}$

Based on equations (5) and (7), the dynamic equations of amplitude and phase are derived as:

$$
\begin{aligned}
& \dot{A}=-\frac{A \sin (2 \beta)}{2 \omega}\left(\omega_{0}^{2}-\omega^{2}\right)-\frac{\omega_{0}^{2}}{\omega k} \sin (\beta)\left(F_{t s}+F_{d}\right)-\frac{A \omega_{0}}{2 Q}(1-\cos (2 \beta)) \\
& \dot{\phi}=-\frac{\cos ^{2}(\beta)}{2 \omega}\left(\omega_{0}^{2}-\omega^{2}\right)-\frac{\omega_{0}^{2}}{A \omega k} \cos (\beta)\left(F_{t s}+F_{d}\right)-\frac{\omega_{0}}{Q} \sin (2 \beta)
\end{aligned}
$$

The transient behaviour of amplitude and phase can be described by the equations (8) and (9).

Integrating equations (8) and (9) in a period of oscillation gives:

$$
\begin{aligned}
& \dot{A}=-\frac{A \omega_{0}}{2 Q}+\frac{F_{0} \omega_{0}^{2} \sin \phi}{2 k \omega}-\frac{\omega_{0}^{2}}{2 \pi k \omega^{2}} \int_{0}^{2 \pi} F_{t s} \sin \beta d \beta \\
& \dot{\phi}=-\frac{1}{2 \omega}\left(\omega_{0}^{2}-\omega^{2}\right)-\frac{F_{0} \omega_{0}^{2} \cos \phi}{2 A k \omega}-\frac{\omega_{0}^{2}}{2 A \pi k \omega^{2}} \int_{0}^{2 \pi} F_{t s} \cos \beta d \beta
\end{aligned}
$$

The nonlinear tip-sample forces are captured by the following functional:

$$
e_{t s}=\frac{1}{\pi} \int_{0}^{2 \pi} F_{t s} \sin \beta d \beta
$$


$v_{t s}=-\frac{1}{\pi} \int_{0}^{2 \pi} F_{t s} \cos \beta d \beta$

where $e_{t s}$ is the energy dissipated during the tip-sample interaction and $v_{t s}$ is the virial of the tip-sample interaction [8], [9], [48].

$\dot{A}=-\frac{A \omega_{0}}{2 Q}+\frac{F_{0} \omega_{0}^{2} \sin \phi}{2 k \omega}-\frac{\omega_{0}^{2}}{2 k \omega} e_{t s}$

$\dot{\phi}=-\frac{1}{2 \omega}\left(\omega_{0}^{2}-\omega^{2}\right)-\frac{F_{0} \omega_{0}^{2} \cos \phi}{2 A k \omega}+\frac{\omega_{0}^{2}}{2 A k \omega} v_{t s}$

By defining $\sigma=\frac{\omega_{0}^{2}-\omega^{2}}{\omega}$ in the steady state, equations (14)-(15) are converted to:

$$
\begin{aligned}
& \frac{A \omega_{0}}{Q}-\frac{A_{0} \omega_{0}^{2} \sin \phi}{k \omega}=-\frac{\omega_{0}^{2}}{2 k \omega^{2}} e_{t s} \\
& \sigma+\frac{A_{0} \omega_{0}^{2} \cos \phi}{A k \omega}=\frac{\omega_{0}^{2}}{A k \omega} v_{t s}
\end{aligned}
$$

Based on the equations (16) and (17) and after some mathematical calculations, the following equations for the steady state amplitude and phase are obtained:

$$
\begin{aligned}
& A=\frac{\left(\frac{v_{t s} k \omega \sigma}{\omega_{0}^{2}}-\frac{e_{t s} k \omega}{\omega_{0} Q}\right)+\sqrt{\left(\frac{e_{t s} k \omega}{\omega_{0} Q}-\frac{v_{t s} k \omega \sigma}{\omega_{0}^{2}}\right)^{2}-\left(\left(\frac{k \omega}{\omega_{0} Q}\right)^{2}+\left(\frac{k \omega \sigma}{\omega_{0}^{2}}\right)^{2}\right)\left(e_{t s}^{2}+v_{t s}^{2}-\left(\frac{k A_{0}}{Q}\right)^{2}\right)}}{\left(\left(\frac{k \omega}{\omega_{0} Q}\right)^{2}+\left(\frac{k \omega \sigma}{\omega_{0}^{2}}\right)^{2}\right)} \\
& \operatorname{tg}(\phi)=\frac{e_{t s}+\frac{k A \omega}{\omega_{0} Q}}{v_{t s}-\frac{k A \omega \sigma}{\omega_{0}^{2}}}
\end{aligned}
$$

From equations (18)-(19), the sensitivity of amplitude and phase to the dissipative and conservative parts of interaction force can be calculated:

$$
\frac{\partial A}{\partial v_{t s}}=\frac{k \omega \sigma}{\omega_{0}^{2}}-\frac{\left(\frac{k \omega \sigma}{\omega_{0}^{2}}\left(\frac{e_{t s} k \omega}{\omega_{0} Q}-\frac{v_{t s} k \omega \sigma}{\omega_{0}^{2}}\right)+\left(\left(\frac{k \omega}{\omega_{0} Q}\right)^{2}+\left(\frac{k \omega \sigma}{\omega_{0}^{2}}\right)^{2}\right) v_{t s}\right)}{\sqrt{\left(\frac{e_{t s} k \omega}{\omega_{0} Q}-\frac{v_{t s} k \omega \sigma}{\omega_{0}^{2}}\right)^{2}-\left(\left(\frac{k \omega}{\omega_{0} Q}\right)^{2}+\left(\frac{k \omega \sigma}{\omega_{0}^{2}}\right)^{2}\right)\left(e_{t s}^{2}+v_{t s}^{2}-\left(\frac{k A_{0}}{Q}\right)^{2}\right)}}
$$


$\frac{\partial A}{\partial e_{t s}}=\frac{-\frac{k \omega}{\omega_{0} Q}+\frac{\left(\frac{k \omega}{\omega_{0} Q}\left(\frac{e_{t s} k \omega}{\omega_{0} Q}-\frac{v_{t s} k \omega \sigma}{\omega_{0}^{2}}\right)-\left(\left(\frac{k \omega}{\omega_{0} Q}\right)^{2}+\left(\frac{k \omega \sigma}{\omega_{0}^{2}}\right)^{2}\right) e_{t s}\right)}{\sqrt{\left(\frac{e_{t s} k \omega}{\omega_{0} Q}-\frac{v_{t s} k \omega \sigma}{\omega_{0}^{2}}\right)^{2}-\left(\left(\frac{k \omega}{\omega_{0} Q}\right)^{2}+\left(\frac{k \omega \sigma}{\omega_{0}^{2}}\right)^{2}\right)\left(e_{t s}^{2}+v_{t s}^{2}-\left(\frac{k A_{0}}{Q}\right)^{2}\right)}}}{\left(\left(\frac{k \omega}{\omega_{0} Q}\right)^{2}+\left(\frac{k \omega \sigma}{\omega_{0}^{2}}\right)^{2}\right)}$

$\frac{\partial \phi}{\partial v_{t s}}=-\frac{\left(e_{t s}+\frac{k A \omega}{\omega_{0} Q}\right)}{\left(v_{t s}-\frac{k A \omega \sigma}{\omega_{0}^{2}}\right)^{2}} \cos ^{2}(\phi)$

$\frac{\partial \phi}{\partial e_{t s}}=\frac{\cos ^{2}(\phi)}{\left(v_{t s}-\frac{k A \omega \sigma}{\omega_{0}^{2}}\right)}$

Note that because of the change of the sign of the force in the attractive and repulsive parts of interaction, in the analyses of sensitivity based on equations (19)-(23), the absolute values are considered.

\section{Numerical Analysis}

In order to analyse the dynamic behaviour and sensitivity of amplitude and phase, several numerical simulations have been performed. The parameters of simulations are as follow:

$f_{0}=300 \mathrm{KHz}, Q=200, k=20 \mathrm{~N} / \mathrm{m}, R=5 \mathrm{~nm}, \mathrm{H}=1 \times 10^{-19} \mathrm{~J}, a_{0}=.164 \mathrm{~nm}, E=1 \mathrm{GPa}$

In Fig.2, the amplitude and phase versus distance curves are shown. These curves are obtained by numerical calculation of equations (8) and (9) for various average distances.

Figs.3-5 show the tip displacement, amplitude and phase versus time, when the cantilever oscillates far from the sample, in the attractive part and in the repulsive part, respectively.
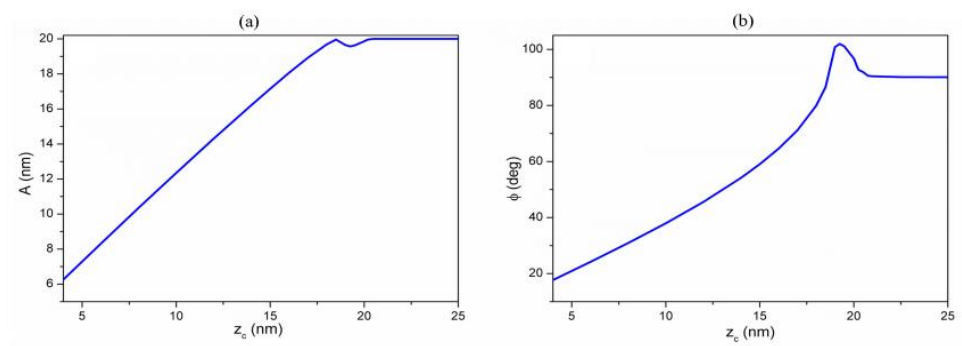

Fig.2. (a) Amplitude versus average tip surface distance (b) Phase versus average tip surface distance. 
(a)

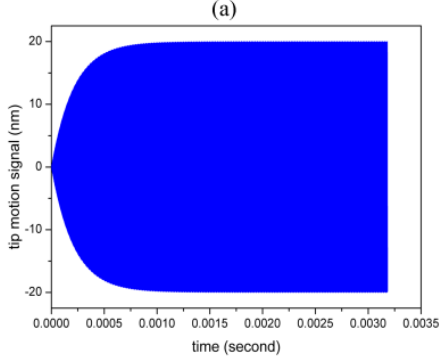

(b)

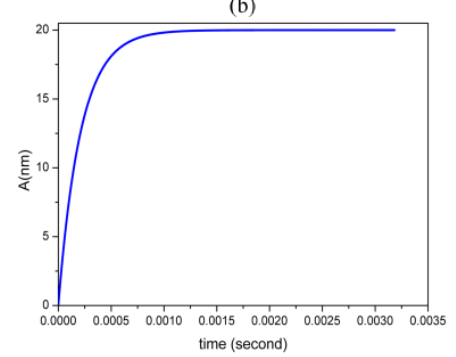

(c)

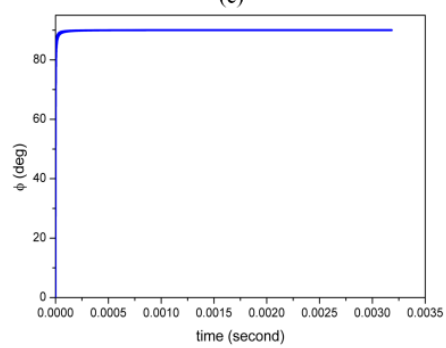

Fig.3. Transient and steady state response of the cantilever-tip system far from the sample, (a) tip motion signal versus time (b) amplitude versus time (c) phase versus time.

(a)

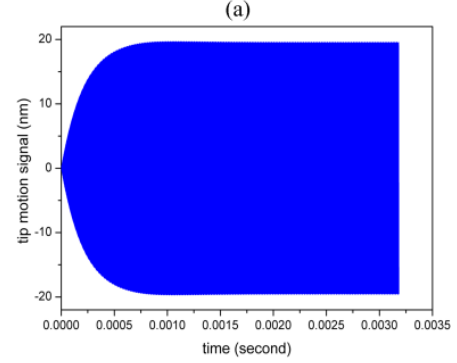

(b)

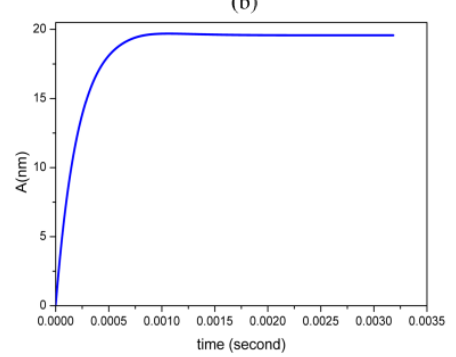

(c)

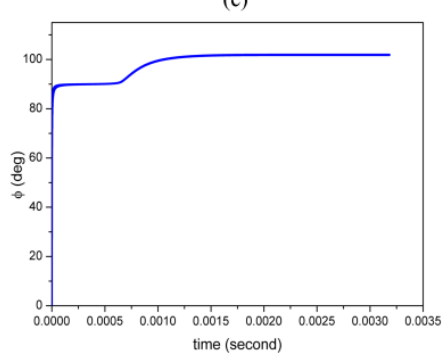

Fig.4. Transient and steady state response of the cantilever-tip system in the attractive regime $\left(z_{c}=19.5 \mathrm{~nm}\right)$, (a) tip motion signal versus time (b) amplitude versus time (c) phase versus time.

(a)

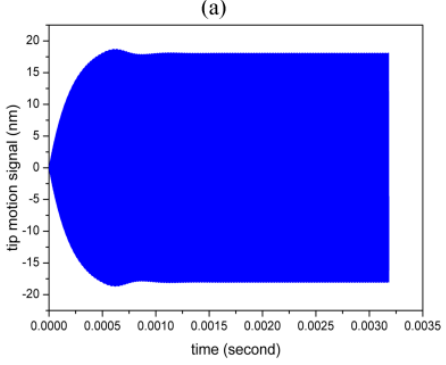

(b)

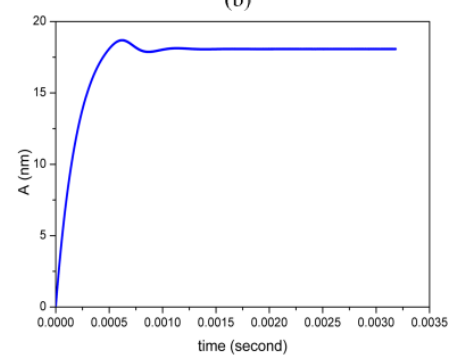

(c)

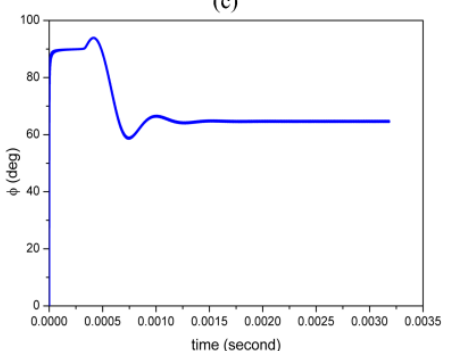

Fig.5. Transient and steady state response of the cantilever-tip system in the repulsive regime $\left(z_{c}=16 \mathrm{~nm}\right)$, (a) tip motion signal versus time (b) amplitude versus time (c) phase versus time.

When the cantilever is far from the sample, the transient response of amplitude to reach the steady state value is much larger than phase. However when there is an interaction between the tip and sample, the transient time of phase is greater than amplitude. In the attractive regime, at first, the phase reaches to 90 degree and after some oscillation periods it goes toward its steady state value. In the repulsive regime, the phase has three different transient behaviours before it reaches to the steady state value. Such as attractive part, at first it goes toward 90 degree, then it has the value more than 90 which is related to the attractive regime, after that it has the value less than 90 and finally it reaches its steady state value in the repulsive part.

All of these behaviours can be explained based on the force-curve analysis. When the tip is far from the sample, there is not any interaction and the cantilever behaves as simple harmonic oscillator. During an approach-retract curve, the cantilever-tip oscillator experiences both a pure attractive and an attractiverepulsive regimes. When the tip is in the pure attractive interaction with the sample, at first the phase goes to 90 and by stabilizing the attractive interaction; it reaches its steady state value which is greater than 90 degree. For repulsive interaction, because before contact with the sample, the tip is in the attractiverepulsive interaction with the sample, at first, the phase goes toward 90 degree then its value goes higher 
than 90 degree which means that the tip has an attractive interaction with the sample, then after experiencing short transient periods in the repulsive part, the phase reaches to its steady state value which is smaller than 90 degree.

To study the effect of conservative and dissipative parts of interaction on the sensitivity of amplitude and phase, different values of Young Modulus and viscosity are considered. In this case the value of free amplitude and Hamaker constant are considered as: $A_{0}=10 \mathrm{~nm}$ and $H=1 \times 10^{-20} \mathrm{~J}$.

The sensitivity of amplitude to the conservative part of interaction (Virial) for different Young Modulus is given in Fig.6a. From this figure it is depicted that for the repulsive regime, decreasing the set-point amplitude leads to the increase of sensitivity. Moreover, the sensitivity is increased by increasing the Young Modulus.
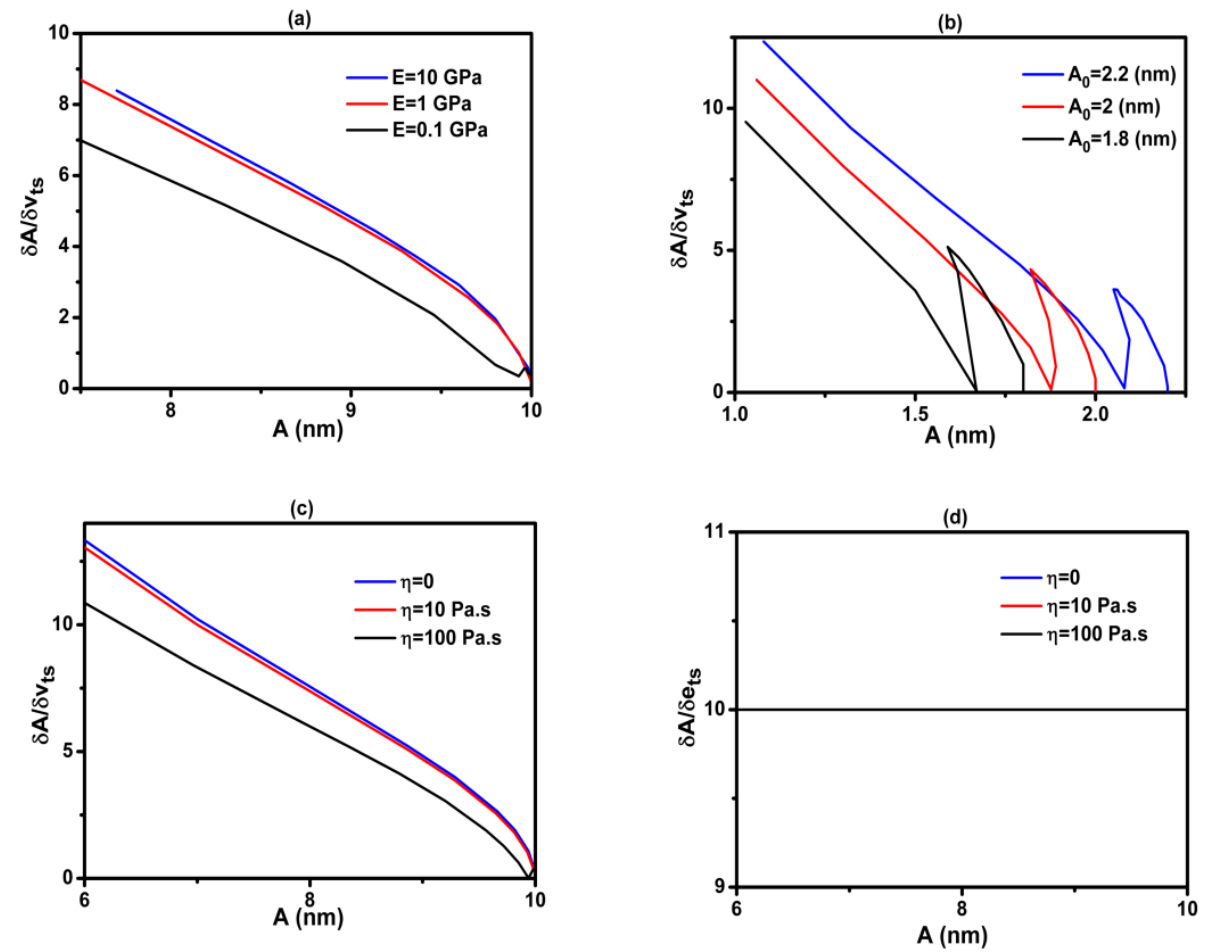

Fig.6. Sensitivity of amplitude to the interaction force, (a) sensitivity of amplitude to the conservative part of interaction for different Young-Modulus, (b) sensitivity of amplitude to the conservative part of interaction for different free amplitudes, (c) sensitivity of amplitude to the conservative part of interaction for different viscosity, (d) sensitivity of amplitude to the dissipative part of interaction for different viscosity.

The effect of free amplitude on the sensitivity is shown in Fig6b. In this case, increasing the free amplitude leads to the increase of sensitivity in the repulsive regime. However, the maximum sensitivity of attractive part can be obtained by decreasing the free amplitude. Moreover, for the attractive interaction, there is a maximum which is increased by decreasing free amplitude. The effect of viscosity on the sensitivity of amplitude to the virial is shown in Fig.6c. The results show that increasing the viscosity, reduces the sensitivity of amplitude to the conservative part of interaction. In Fig. $6 \mathrm{~d}$ the sensitivity of amplitude to the dissipation is shown. It is clear that the dissipation has not any impact in the sensitivity of amplitude.

In Fig.7a,b the difference of phase shift for different young modulus and viscosity is shown. For higher viscosity at the same young modulus and amplitude, the phase is greater while for less young modulus, in the case of same viscosity and same amplitude, the phase is higher. 
Fig.7c,d shows the results of the sensitivity of phase to the virial and dissipation for the different young modulus while the viscosity is constant. From the results it is depicted that increasing the stiffness leads to the increase of phase sensitivity to dissipation and decrease of phase sensitivity to virial.

Fig.7e,f shows the sensitivity of phase to the virial and dissipation in the case of constant young modulus and different viscosity. Results show that increasing the viscosity leads to the increase of phase sensitivity to virial and decrease of that to the dissipation.

To explain this behaviour, we can refer to the origin of phase contrast. As [2]-[4] explain, the source of phase contrast is related to the dissipation. However, for hard materials, in some cases, the higher harmonics also have contribution [15], [50]. So, for the same young modulus and amplitude as it is shown in Fig. 7b, the variation of phase versus to 90 degree, is decreased by increasing the viscosity, and in other hand, the dissipation is increased by increasing the viscosity, hence the variation of phase to dissipation is reduced. In contrast, for the case that viscosity is kept constant and young modulus is changed, as Fig.7a shows, the variation of phase in versus to 90 degree is increased by increasing the young modulus, on the other hand increasing the young modulus leads to the decrease of dissipation, therefore, increasing the young modulus leads to the increase of phase sensitivity to dissipation. Moreover, by reduction of amplitude the sensitivity of phase to the dissipation is increased while the sensitivity of phase to conservative part of interaction is decreased.

(a)

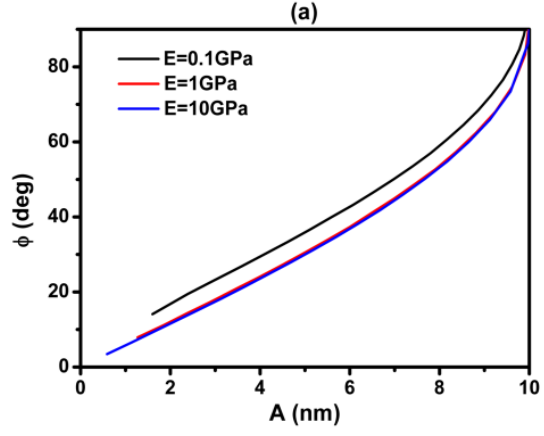

(c)

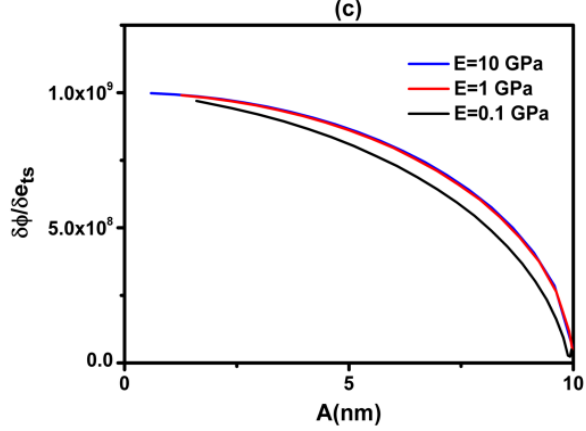

(e)

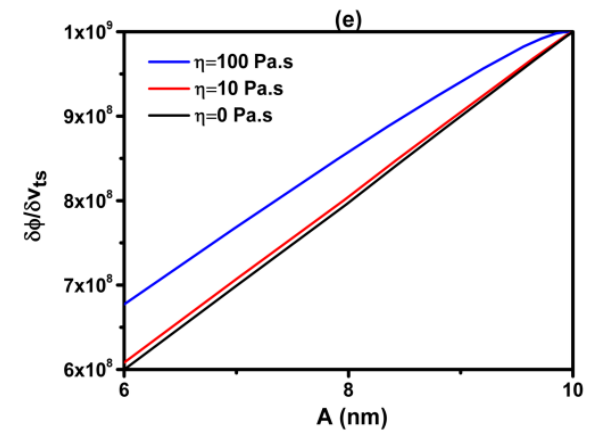

(b)

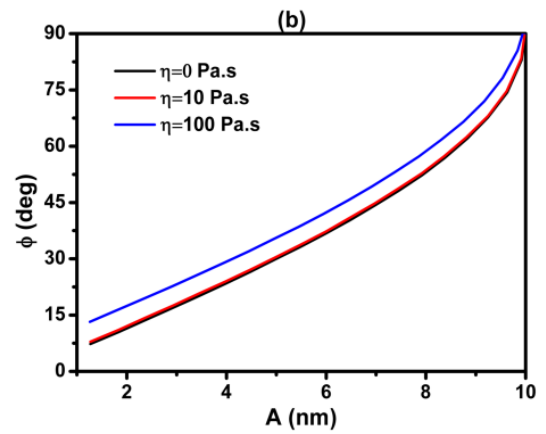

(d)

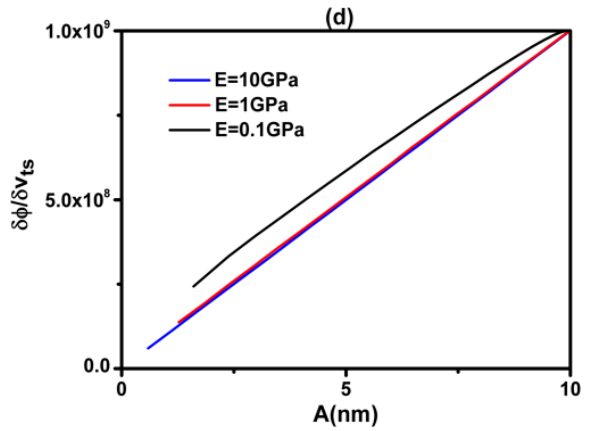

(f)

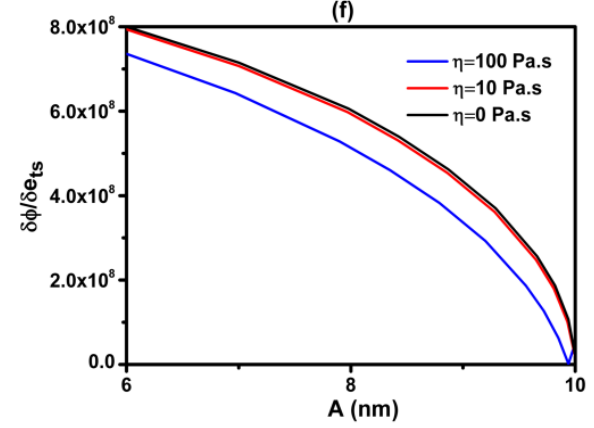

Fig.7. Sensitivity of phase to the interaction force, (a) phase versus amplitude for different stiffness, (b) phase versus amplitude for different viscosity (c) sensitivity of phase to the dissipative part of interaction for different Young-Modulus, (d) sensitivity of phase 
to the conservative part of interaction for different Young-Modulus, (e) sensitivity of phase to the conservative part of interaction for different viscosity, (d) sensitivity of phase to the dissipative part of interaction for different viscosity.

\section{Conclusion}

In this paper, the slow time varying function theory is used to model the dynamics equations of amplitude and phase of the atomic force microscopy. The advantage of using this model is the ability to simulate and analysis the dynamics behavior of amplitude and phase of the AFM tip motion not only in the steady state but also in the transient regime. Then, the sensitivity of amplitude and phase to the conservative and dissipative parts of interaction by considering different young modulus, viscosity, free and set-point amplitudes are analyzed. This analysis gives deep and comprehensive information about the relation between parameters of oscillation and interaction.

\section{Acknowledgements}

The author gratefully acknowledge Prof. Ricardo Garcia from the Instituto de Ciencia de Materiales de Madrid for his encouraging comments and supports.

\section{References}

[1] R. García and A. San Paulo, Phys. Rev. B, 1999, 60, 4961.

[2] J. Tamayo and Ricardo García, Appl. Phys. Lett., 1998, 73, 2926.

[3] J. P. Cleveland, B. Anczykowski, A. E. Schmid, and V. B. Elings, Appl. Phys. Lett., 1998, 72, 6961.

[4] R. García, J. Tamayo, and A. San Paulo, Surf. Interface Anal., 1999, 27, 312.

[5] R. García and A. San Paulo, Phys. Rev. B, 2000, 61, R13381.

[6] A. San Paulo and R. García, Biophys. J., 2000, 78, 1599.

[7] R. García and A. San Paulo, Ultramicroscopy, 2000, 82, 79.

[8] A. San Paulo and R. García, Phys. Rev. B, 2001, 64, 193411.

[9] B. Anczykowski, B. Gotsman, H. Fuchs, J. P. Cleveland, and V. B. Elings, Appl. Surf. Sci., 1999, $140,376$.

[10] A. San Paulo and R. García, Surf. Sci., 2001, 471, 71.

[11] R. García, C.J. Gómez, N.F. Martínez, S. Patil, C. Dietz, and R. Magerle, Phys. Rev. Lett., 2006, 97, 016103-4.

[12] L. N. Kantorovich and T. Trevethan, Phys. Rev. Lett., 2004, 93, 236102.

[13] M. Stark, C. Moller, D. J. Muller, and R. Guckenberger, Biophys J., 2001, 80(6), 3009-3018.

[14] S. Santos, V. Barcons, A. Verdaguer, J. Font, N. H. Thomson, and M. Chiesa, Nanotechnology, 2011, 22, 345401 .

[15] A. F. Payam, J. R. Ramos, and Ricardo García, ACS Nano, 2012, 6, 4663-4670.

[16] C. A. Amadei, T. C. Tang, M. Chiesa, et ál., J. Chem. Phys., 2013, 139(8), 084708.

[17] S. Santos, V. Barcons, H. K. Christenson, et ál., Appl. Phys. Lett., 2013, 103, 063702.

[18] D. Kiracofe, and A. Raman, J. Appl. Phys., 2010, 107, 033506.

[19] S. Santos, C. A. Amadei, A. Verdaguer, et ál., J. Chem. Phys., 2013, 117(20), 10615-10623.

[20] K. R. Gadelrab, S. Santos, and M. Chiesa, Langmuir, 2013, 29(7), 2200-2206.

[21] S. Santos, K. R. Gadelrab, V. Barcons, et ál., J. Appl. Phys., 2012, 112(12), 124901.

[22] M. Gauthier, R. Pérez, T. Arai, M. Tomitori, and M. Tsukada, Phys. Rev. Lett., 2002, 89, 146104.

[23] R. Garcia, Amplitude Modulation Atomic Force Microscopy, (Wiley-VCH, Weinheim, 2010).

[24] J. M. Balthazar, A. M. Tusset, S. L. Thomaz de Souza, and A. M. Bueno, J. Mech. Eng. Sci., 2012, Part C, 0954406212467933.

[25] A. Passian, A.L. Lereu, D. Yi, S. Barhen, and T. Thundat, Phys. Rev. B, 2007, 75, 233403.

[26] A. Passian, G. Muralidharan, A. Mehta, H. Simpson, T. L. Ferrell, and T. Thundat, Ultramicroscopy, 2003, 97, 391-399.

[27] K. R. Gaderlab, S. Santos, J. Font, and M. Chiesa, Nanoscale, 2013, 5(22), 10776-10793.

[28] S. Santos, K. R. Gaderlab, J. Font, and M. Chiesa, New J. Phys., 2013, 15, 083034.

[29] D. R. Sahoo, Transient force atomic force microscopy: systems approaches to emerging applications, $\mathrm{PhD}$ Thesis, (Iowa State University, 2006).

[30] D. R. Sahoo, A. Sebastian, and M. V. Salapaka, Appl. Phys. Lett., 2003, 83(26), 5521-5523.

[31] D. R. Sahoo, A. Sebastian, and M. V. Salapaka, Int. J. Robust Nonlinear Control, 2005, 15,805-820.

[32] P. I. Chang, P. Huang, J. Maeng, and S. B. Andersson, Rev. Sci. Instrum., 2011, 82, 063703.

[33] P. Huang and . B. Andersson, American control conference, Farimont queen Elizabeth, Montreal, Canada, June 27-June 29, 2012.

[34] J. A. Turner and J. S. Wiehm, Nanotechnology, 2001, 12, 322-330.

[35] W. J. Chang, Nanotechnology, 2002, 13, 510-514.

[36] A. F. Payam, Scientia Iranica B, 2013, 20(4), 1221-1227.

[37] A. F. Payam, Ultramicroscopy, 2013, 135, 84-88.

[38] A.F. Payam, M.Fathipour, Int J Adv Manuf Technol., 2013, 65, 957-966.

[39] T. M. Arruda, A. Kumar, S.V. Kalinin, and S. Jesse, Nano Lett., 2011, 11, 4161-4167. 
[40] R. M. Brydson, C. Hammond, D. Mowbray, M. R. J. Gibbs, I. Todd, et al., Nanoscale Science and Technology, (Wiley, Chichester, 2005).

[41] O. Sahin, S. Magonov, C. Su, C. F. Quate and O. Solgaard, Nat. Nanotechnol., 2005, 2, 507-514.

[42] D. Platz, E. A. Tholen, D. Pesen, and D. B. Haviland, Appl. Phys. Lett., 2008, 92, 153106-153108.

[43] T. Rodriguez and R. Garcia, Appl. Phys. Lett., 2004, 84, 449- 551.

[44] S. Kawai, T. Glatzel, S. Koch, B. Such, et al., Phys. Rev. Lett., 2009, 103, 220801-220804.

[45] R. Garcia and E. T. Herruzo, Nat. Nanotechnol., 2012, 7, 217- 226.

[46] L. Nony, R. Boisgard, J. P. Aime, J. Chem. Phys., 1999, 111(4), 1615-1627.

[47] A. Sinha, J. Sound Vib. 288, 387-394 (2005).

[48] J. Melcher, D. Martinez-Martin, M. Jaafar, J. G. Herrero, and A. Raman, Beilstein J Nanotechnol., 2013, 4, 153 163.

[49] A. F. Payam, D. M. Jimenez, R. Garcia, Nanotechnology, 2015, 26.

[50] J. Tamayo, Appl. Phys. Lett., 1999, 75, 3569-3571. 